\title{
Olfactory and gustatory functions in patients with non-complicated Type1 Diabetes Mellitus
}

Seyid Ahmet AY ${ }^{1}$, Serdar HiRA 2 , Murat SALIHOGLU ${ }^{3}$, Ferhat DENIZ ${ }^{1}$, Aytug ALTUNDAG ${ }^{4}$, Kamil BASKOY ${ }^{1}$, Hakan TEKELI ${ }^{5}$, Arif YONEM ${ }^{1}$, Thomas Hummel ${ }^{6}$ 1.Dep. of Endocrinology, Haydarpaşa Training Hospital, GATA, Istanbul; 2. Dep. of Biochemistry, Haydarpaşa Training Hospital, GATA, Istanbul; 3.Dep. of Otorhinolaryngology, Haydarpaşa Training Hospital, GATA, Istanbul; 4.Dep. of Otorhinolaryngology, Istanbul Surgery Hospital, Istanbul; 5.Dep. of Neurology, Haydarpaşa Training Hospital, GATA, Istanbul; 6.Dep. of Otorhinolaryngology, TU Dresden

Introduction: Olfactory and gustatory dysfunction in patient with Diabetes mellitus(DM) and its pathophysiology were studied in many previous studies. Some studies reported that there was a relationship between Type1 DM(T1DM) and olfactory and gustatory functions the presence of diabetic complications. However findings are limited and controversial. The aim of this study was to determine the relationship between olfactory and gustatory scores and patients with non-complicated T1DM. Another aim was to present evidence of the association between olfactory and gustatory scores and $\mathrm{HbA1c}$ values and disease durations.

Methods: The study included 39 out-patient non-complicated T1DM patients and 31 healthy individuals. Psychophysical olfactory tests were performed using the commercially available "Sniffin' Stick" test kit. Taste function tests were carried out using "Taste Strips" method.

\begin{tabular}{lccc} 
Table 2. Olfactory and taste results of patients and controls \\
$\begin{array}{lccc}\text { Control Group } \\
(\mathbf{n}=\mathbf{3 1})\end{array}$ & $\begin{array}{c}\text { Type 1 Diabetes } \\
\text { Mellitus patients } \\
(\mathbf{n}=39)\end{array}$ & p value \\
\hline Threshold & $8,63 \pm 0,91$ & $8,55 \pm 0,57$ & 0,66 \\
Discrimination & $12,97 \pm 0,80$ & $12,74 \pm 0,79$ & 0,24 \\
Identification & $13,81 \pm 0,98$ & $13,72 \pm 0,89$ & 0,69 \\
TDI scores & $35,34 \pm 1,94$ & $34,97 \pm 1,40$ & 0,37 \\
Bitter & $3,45 \pm 0,51$ & $3,44 \pm 0,50$ & 0,90 \\
Sweet & $3,32 \pm 0,48$ & $3,38 \pm 0,49$ & 0,60 \\
Salt & $3,13 \pm 0,72$ & $3,10 \pm 0,72$ & 0,88 \\
Sour & $3,26 \pm 0,58$ & $3,26 \pm 0,60$ & 0,99 \\
Total score taste & $13,16 \pm 1,61$ & $13,13 \pm 1,22$ & 0,92 \\
\hline \multicolumn{4}{l}{} \\
\hline
\end{tabular}

Results: There were no significant differences in olfactory tests between two groups (odor thresholds: $8.63 \pm 0.91$ vs $8.55 \pm 0.57, p=0.66$; odor discrimination: $12.97 \pm 0.80$ vs $12.74 \pm 0.79, p=0.24$; odor identification: $13.81 \pm 0.98$ vs 13.72 $\pm 0.89, p=0.69$; TDI score: $35.34 \pm 1.94$ vs $34.97 \pm 1.4, p=0.37)$. There were also no significant differences in gustatory tests between two groups (bitter: $3.45 \pm 0.51$ vs $3.44 \pm 0.50, p=0.90$; sweet: $3.32 \pm 0.48$ vs $3.38 \pm 0.49$, $p=0.60$; salty: $3.13 \pm 0.72$ vs $3.10 \pm 0.72, p=0.88$; total score of taste: $13.16 \pm 1.61$ vs $13.13 \pm 1.22, p=0.92)$. When dividing T1DM patients according to HbA1c values into three subgroups 1) HbA1c values<7; 2) HbA1c values 79; 3) $\mathrm{HbA} 1 \mathrm{c}$ values $>9$, olfactory tests and gustatory tests did not differ between groups ( $>0.05$ ). Comparison of gustatory and olfactory scores according to disease duration revealed that there were no differences between groups $(p>0.05)$.

Conclusion: This study demonstrates that T1DM without complications is not associated with olfactory and gustatory dysfunction. We also found that gustatory and olfactory functions are not related with HbA1c values and disease duration. Further research is needed with regard to the underlying mechanisms to explain olfactory and gustatory dysfunction in T1DM with complications. 